\title{
EJEMPLARES DE LA PHYLLOPOD BED-BURGESS SHALE (CÁMBRICO MEDIO) EN EL MUSEO NACIONAL DE CIENCIAS NATURALES-CSIC (MADRID, ESPAÑA)
}

\author{
Ángel MONTERO', Carmen DIÉGUEZ' y Diego GARCÍA-BELLIDO' \\ ' Museo Nacional de Ciencias Naturales-C.S.I.C. José Gutiérrez Abascal, 2. E-28006 Madrid. España. \\ `Depto. de Paleontología. Fac. de Ciencias Geológicas. Universidad Complutense. E-28040 Madrid. España. \\ Correo electrónico: diegogbc@eucmax.sim.ucm.es
}

\begin{abstract}
Montero, Á., Diéguez, C. y García-Bellido, D. 1998. Ejemplares de la Phyllopod Bed-Burgess Shale (Cámbrico Medio) en el Museo Nacional de Ciencias Naturales-CSIC (Madrid, España). [Burgess Shale specimens from the Phyllopod Bed (Middle Cambrian) at the Museo Nacional de Ciencias Naturales-CSIC (Madrid, Spain)]. Revista Española de Paleontología, n⿳0 extr. Homenaje al Prof. Gonzalo Vidal, 143-148. ISSN 0213-6937.
\end{abstract}

\begin{abstract}
A collection of fossil invertebrates from well-known North American sites is hosted at the Museo Nacional de Ciencias Naturales-CSIC (MNCN-CSIC) of Madrid. This collection was a result of an exchange between this Institution and the Smithsonian Institution of Washington in the sixties of this century. In this collection there are eigth items from the Walcott's Quarry site of the Burgess Shale (British Columbia, Canada). It consists of twelve specimens included in the Kingdom Monera (one pliy/um) and Kingdom Animalia (six phy/a).
\end{abstract}

Key words: Exchange, Smithsonian Institution, MNCN-CSIC, fossils, Middle Cambrian, Burgess Shale.

\section{RESUMEN}

El Museo Nacional de Ciencias Naturales-CSIC (MNCN-CSIC), conserva entre sus fondos una colección de invertebrados fósiles de yacimientos típicos norteamericanos, consecuencia de un intercambio en los años sesenta de este siglo con la Smithsonian Institution de Washington. Dentro de esta colección se encontraba una muestra bastante representativa de fauna fósil del yacimiento de Walcott's Quary de la Burgess Shale (Columbia Británica, Canadá), consistente en ocho muestras de mano conteniendo doce especies de seis phyla del Reino Animalia y un phylum del Reino Monera.

Palabras clave: Intercambio, Smithsonian Institution, MNCN-CSIC, fósiles, Cámbrico Medio, Burgess Shale.

\section{INTRODUCCIÓN}

En la década de los sesenta del presente siglo, el Museo Nacional de Ciencias Naturales-CSIC (MNCNCSIC) llevó a cabo un intercambio de fósiles con la Smithsonian Institution de Washington (B. Meléndez, com. pers., 1994), mediante el cual llegaron al Museo ciento ochenta y ocho ejemplares de localidades típicas norteamericanas paleozoicas y cenozoicas: Cámbrico de Antelope Springs en Utah, Silúrico de Louisville en Kentucky, Devónico del Lago Erie en Nueva York, Carbonífero de Crawforsville en Indiana o de Jacksboro en Texas, Plioceno de Shell Creek en Florida y otras.

Entre todo este material se encontraban algunos ejemplares de la Burgess Shale (Canadá), y concretamente de la Phyllopod Bed (2,3 m de potencia), siglados por la institución americana como $35 \mathrm{k}$. Los fósiles de este yacimiento que posee el MNCN están incluidos en lutitas negras micáceas de grano muy fino, matriz típica en la Phyllopod Bed. Dos ejemplares (MNCNI-5220, MNCNI-520lb) presentan una pátina superficial de color pardo debida a meteorización de la lutita en el propio yacimiento.

La colección se encontraba en los años ochenta en la Sala de Exposición permanente de Paleontología, almacenada en un armario, de donde se extrajo para ser inventariada y reubicada junto al resto de colecciones paleontológicas (Montero y Diéguez, 1991a). Todos los 


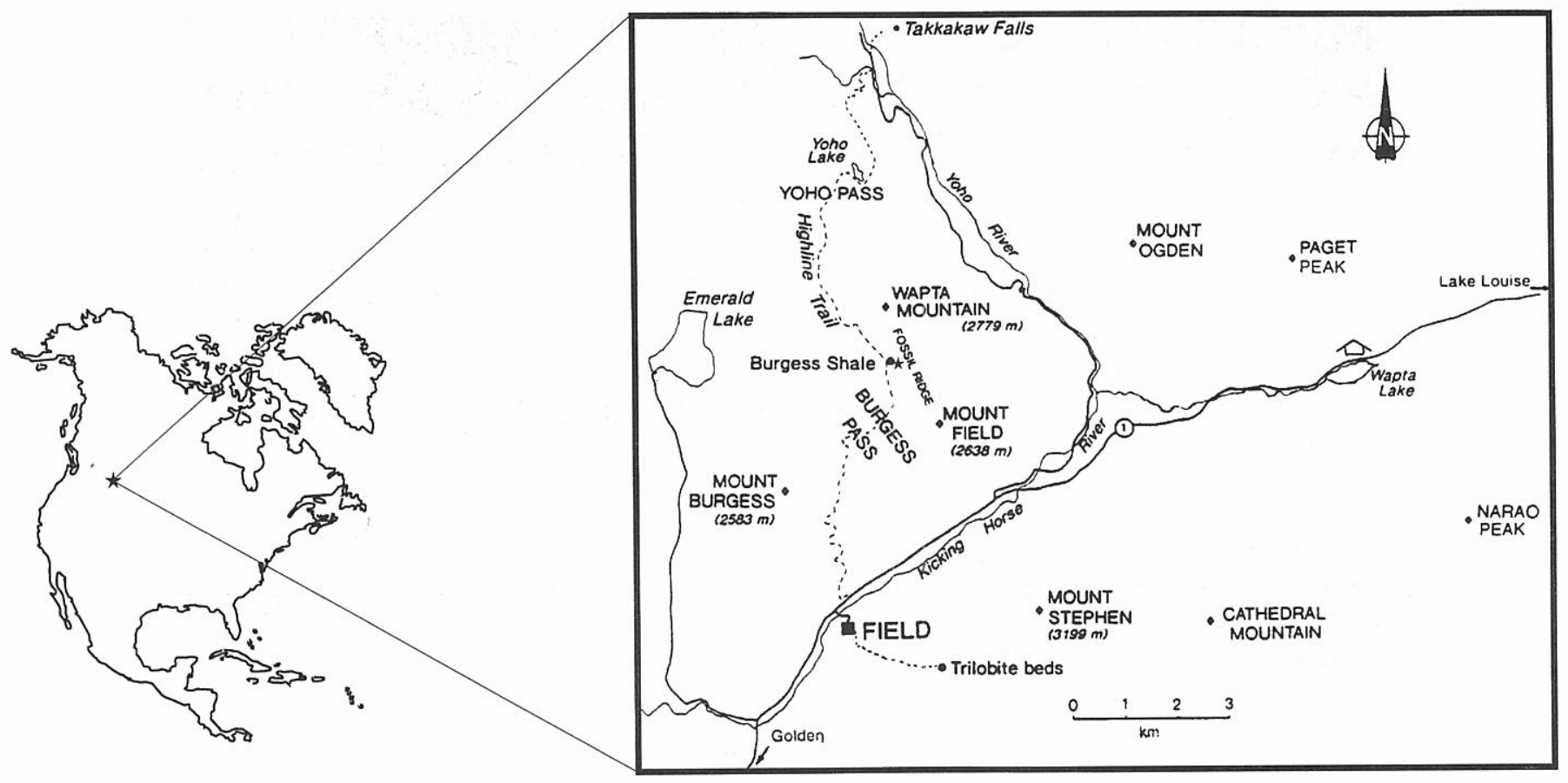

Figura 1. Localización geográfica del yacimiento de Walcott's Quarry-Burgess Shale, Columbia Británica, Canadá. (Modificada de Collins y Steward, 1991.)

ejemplares se encontraban tal y como fueron enviados por la Smithsonian Institution, es decir, incluidos en cajas de cartón sin cobertor y con una base de algodón. El inconveniente de los contenedores abiertos es la acumulación de polvo ambiental y contaminantes, problema más acusado en museos dentro de núcleos urbanos y el del algodón como base es la adherencia a superficies rugosas o fracturadas. El tratamiento dado a los ejemplares consistió en un cambio de contenedor (caja de poliestireno rígido transparente con tapa) y de la base (polietileno de baja densidad y alta presión), al igual que la aplicada al resto de la colección (Diéguez y Montero, 1994; Montero, 1995).

Las etiquetas originales de la Smithsonian Institution aportaban una información completa de todo el material de la colección a excepción de la localidad. Este último dato, necesario en ejemplares de museo (Montero y Diéguez, 1991b), queda aparentemente soslayado, al menos a medio plazo, al provenir los ejemplares de localidades muy conocidas y que se siguen explotando en la actualidad.

En estos momentos el material procedente de la Burgess Shale está a disposición de la comunidad paleontológica para su estudio o consulta, al igual que el resto de la colección del MNCN-CSIC, que ha sido inventariada y almacenada en condiciones adecuadas.

\section{EL YACIMIENTO DE WALCOTT'S QUARRY-BURGESS SHALE}

La localidad tipo de la Burgess Shale se encuentra a $2.300 \mathrm{~m}$ de altitud en la ladera oeste de Fossil Ridge, al Norte del pueblo de Field, en el Parque Nacional de Yoho, Columbia Británica, Canadá (Fig. 1). Este yacimiento fue encontrado en 1909 por Charles Walcott, Secretario de la Smithsonian Institution de Washington, mientras se hallaba estudiando las rocas proterozoicas y cámbricas que afloran en las Montañas Rocosas canadienses, como máximo experto en materiales cámbricos (Yochelson, 1996). Se denomina Burgess Shale a las capas de la Formación Stephen que conservan fósiles de organismos de cuerpo blando. La Formación Stephen (Cámbrico Medio) está constituída por más de $190 \mathrm{~m}$ de lutitas con intercalación a techo de algunos niveles calizos; está limitada a muro por la Formación Cathedral y a techo por la Formación Eldon (Fig. 2). Un año más tarde Walcott localizó la Phyllopod Bed (siglas $35 \mathrm{k}$ en los fósiles), que es el nombre informal que reciben las capas más productivas en cantidad y calidad de fósiles dentro del yacimiento de la Burgess Shale. Estas capas fueron excavadas exhaustivamente por Walcott en el periodo 1910-1913, así como en 1917, 1919 y 1924, proporcionando la mayor colección de fósiles de Burgess

\section{Lámina I.}

Barra de escala $=1 \mathrm{~cm}$

1 Yacimiento de Walcott's Quarry-Burgess Shale durante la campaña de 1995. Se señala el nivel 35k, conocido como Phyllopod Bed. (Tomada por uno de los autores, D. G.-B.)
2 Pagetia bootes Walcott, 1916. (MNCNI-5220.)

3 Marrella splendens Walcott, 1912. (MNCNI-5170.)

4 Vauxia gracilenta Walcott, 1920. (MNCNI-5120.) 


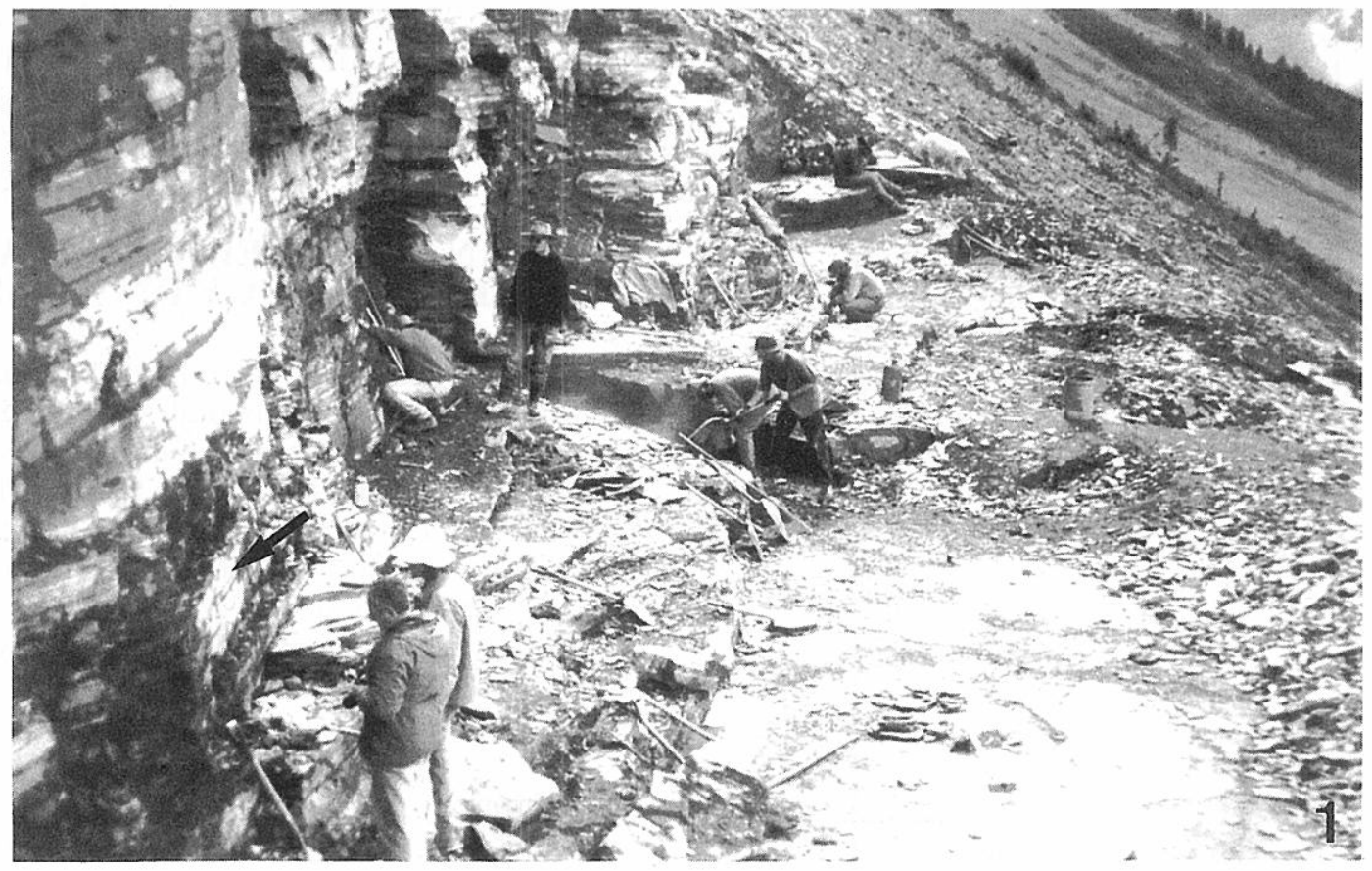

Lámina I
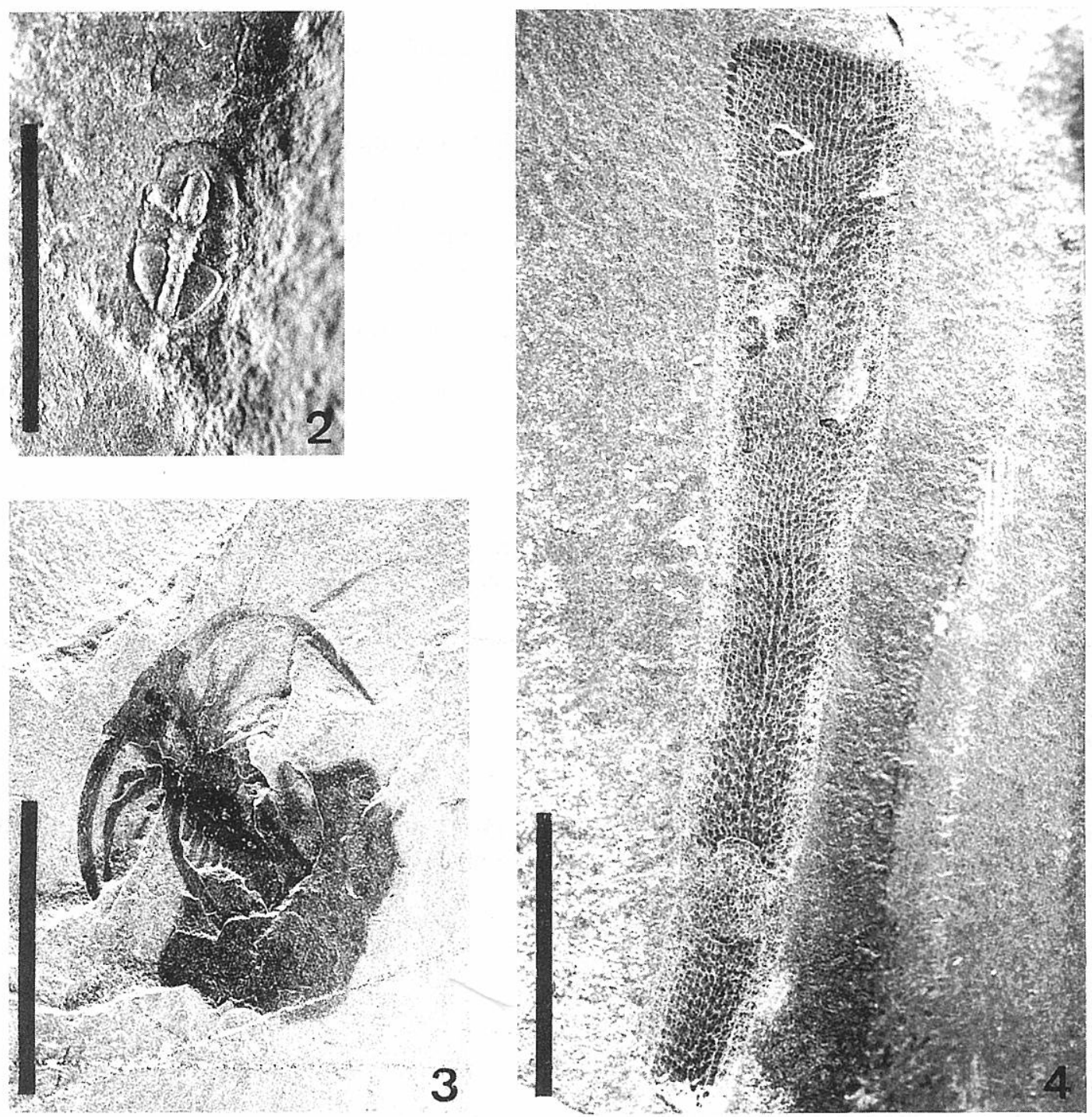

Revista Española de Paleontología, nº extr. Homenaje al Prof. Gonzalo Vidal, 1998. 


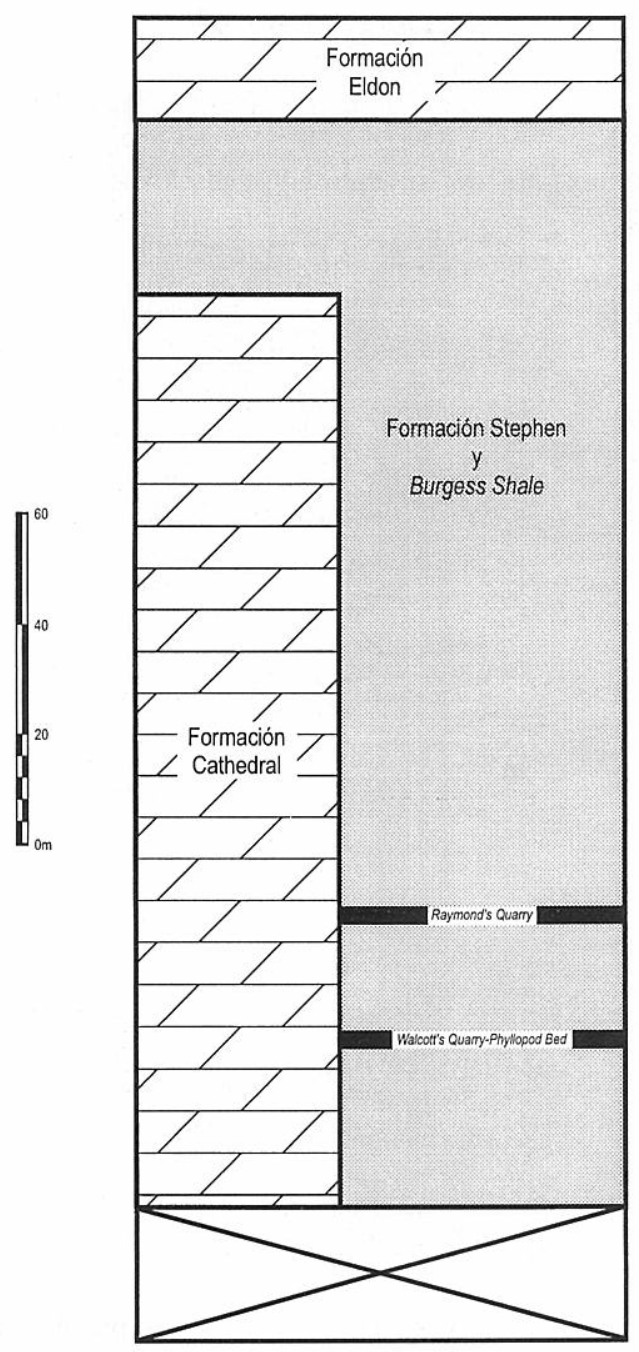

Figura 2. Sección tipo de la Formación Stephen y Burgess Shale. (Modificada de Collins y Steward, 1991.)

Shale, depositada en el National Museum of Natural History-Smithsonian Institution, con cerca de 65.000 ejemplares. Posteriormente, en 1930, trabajó en el yacimiento Percy Raymond, de la Universidad de Harvard, excavando el mismo nivel que Walcott y las capas situadas veinte metros por encima de la Phyllopod Bed (Whittington, 1985).

Ambas excavaciones dejaron marcas patentes en la ladera, constituyendo las canteras conocidas como Walcott's Quarry y Raymond's Quarry. En 1966 y 1967, se organizaron nuevas excavaciones en estas canteras dirigidas por Aitken, Fritz y Whittington. Al terminar las excava-ciones, Whittington comenzó el "Proyecto Cambridge" en el que colaboraron sus colegas Bruton, Hughes, Briggs y Conway Morris, estos dos últimos realizando sus tesis doctorales sobre los fósiles del yacimiento. Desde 1983 y hasta la actualidad, un equipo del Royal Ontario Museum de Toronto, dirigido por Desmond Collins, ha llevado a cabo numerosas campañas en el yacimiento (Lám. I, fig. 1). Por último, diremos que en 1981 la UNESCO, reconociendo el valor incalculable que tiene la localidad tipo de la Burgess Shale, la designó
"Patrimonio Mundial de la Humanidad", con lo que le confiere una figura de protección que la conservará para futuras generaciones (Whittington, 1985; Briggs et al., 1994).

\section{ANÁLISIS DEL MATERIAL. IMPORTANCIA Y DIVERSIDAD}

La representación de la fauna fósil de Burgess que la Smithsonian Institution envió al MNCN-CSIC constaba de ocho piezas que fueron incluidas en seis registros de inventario: MNCNI-4917, MNCNI-5120, MNCNI-5170, MNCNI-5181, MNCNI-5201 (con tres piezas desdobladas por nosotros en MNCNI-5201a, MNCNI5201b y MNCNI-5201c) y MNCNI-5220, presentando algunas piezas un ejemplar individualizado y otras varios (Montero y Diéguez, 1996). La relación de grupos y especies es la siguiente:

\section{Reino Monera}

Phylum Cyanobacteria

Marpolia spissa Walcott, 1919. (MNCNI-5201a y c.) Filamentos. Material abundante en algunos tramos de la Phyllopod Bed y que llega a ser muy escaso en otros.

\section{Reino Animalia}

Phylum Porifera

Clase Demospongea

Vauxia gracilenta Walcott, 1920. (MNCNI-5120.) (Lám. I, fig. 2.) Un ejemplar no ramificado. No aparece frecuentemente. Entre los poríferos es el más común en la capa.

Porifera indet. (MNCNI-5201c.) Espículas.

Phylum Brachiopoda

Clase Articulata.

Diraphora bellicostata (Walcott, 1924). (MNCNI$5201 \mathrm{a}, \mathrm{b}$ y c.) Trece valvas desarticuladas. Especie relativamente frecuente.

Nisusia burgessensis Walcott, 1924. (MNCNI-5220.) Una valva. Material poco común en la Phyllopod Bed.

Brachiopoda indet. (MNCNI-5201c.) Una valva.

Phylum Mollusca

Clase Monoplacophora.

Scenella amii (Matthew, 1902). (MNCNI-5201a y c; MNCNI-4917.) Cuatro ejemplares. Muy abundante en algunos tramos.

Phylum Hyolitha

Haplophrentis carinatus (Matthew, 1899). (MNCNI5201 a y c.) Un ejemplar con opérculo y otro sin él. Relativamente común.

Phylum Priapulida

Selkirkia columbia Conway Morris, 1977. (MNCNI5201c.) Un ejemplar incompleto del tubo. Poco común. 
Phylum Arthropoda

Marrella splendens Walcott, 1912. (MNCNI-5170.) (Lám. I, fig. 3.) Un ejemplar completo. Abundante y representativo de la capa.

Clase Trilobita.

Pagetia bootes Walcott, 1916. (MNCNI-5220.) (Lám. I, fig. 4.) Un ejemplar completo. Poco abundante.

Peronopsis montis (Matthew, 1899). (MNCNI-5181.) Un ejemplar completo. Poco abundante.

Ptychagnostus praecurrens (Westergård, 1936). (MNCNI-5201b y c.) Un cefalón y un pigidio. Poco abundante.

\section{Incertae Sedis}

Wiwaxia corrugata (Mattew, 1899). (MNCNI-5201c.) Un esclerito dorsal largo. Poco común.

\section{CONCLUSIONES}

En el material de la Burgess Shale depositado en el MNCN-CSIC existen un total de 14 taxones y 12 especies. El material descrito en la Burgess Shale consta de 125 géneros y 173 especies (Briggs et al., 1994); por tanto, la colección del MNCN-CSIC supera ligeramente el $8 \%$ de géneros descritos en el yacimiento y el $6 \%$ de especies. Desde el punto de vista sistemático, entre el material depositado en el MNCN-CSIC están presentes ejemplares atribuibles a dos de los tres reinos presentes en la Burgess Shale: Monera, Protista y Animalia. Del Reino Monera aparecen tan solo representantes de una División, en forma de algunos filamentos de Cianobacterias. Del Reino Animalia se tienen seis phyla de los trece descritos en la Burgess Shale, es decir cerca del $50 \%$, y un Incertae Sedis de las veintiuna especies, no asignadas a ningún Phylum, que han aparecido en este yacimiento.

La importancia de la colección depositada en el MNCN-CSIC viene dada por la representatividad del material, ya sea por la presencia de ejemplares abundantes en el yacimiento, como Marrella splendens o Scenella amii, o por la de ejemplares poco abundantes como Selkirkia columbia o Wiwaxia corrugata. Así como por la carencia total de ejemplares de esta fauna fósil en otras instituciones españolas.

Esta colección, tiene interés, también, por pertenecer en su totalidad a la capa que explotó Walcott (Phyllopod $B e d)$, señalados con la sigla $35 \mathrm{k}$. El estudio detallado del material ha proporcionado nuevos ejemplares de cinco especies que no se habían detallado con anterioridad, algunas de manera fragmentaria, ya que la relación que envió la Smithsonian Institution consistía sólo en siete especies, que son las que se citan en un trabajo anterior (Montero y Diéguez, 1996), mientras que la relación que se aporta en este trabajo las eleva a doce, lo que revaloriza considerablemente esta colección. La preparación del material podría, en un futuro, añadir nuevos ejemplares de otras especies a las ya citadas por nosotros.

\section{BIBLIOGRAFÍA}

Briggs, D. E. G., Erwin, D. H. and Collier, F. J. 1994. The Fossils of the Burgess Shale. Smithsonian Institution Press, Washington, London. 238 pp.

Collins, D. H. and Stewart, W. D. 1991. The Burgess Shale and its environmental setting, Fossil Ridge, Yoho National Park. In: A Field Guide to the Paleontology of Southwestern Canada (Ed. P. L. Smith). University of British Columbia, Victoria, 104-118.

Conway Morris, S. 1977. Fossil priapulid worms. Special Papers in Palaeontology, 20, 1-95.

Diéguez, C y Montero, A. 1994. Organización y gestión de los fondos paleontológicos. In: Manual de catalogación y gestión de las colecciones científicas de Historia Natural (Ed. B. Sanchiz). Manuales Técnicos de Museología, vol. 1., Museo Nacional de Ciencias Naturales-CSIC, Madrid, 161-204.

Matthew, G. F. 1899. Studies on Cambrian faunas, no. 3: Upper Cambrian fauna of Mt. Stephen, British Columbia. Transactions of the Royal Society of Canada, Series 2, 5, 3-66.

Matthew, G. F. 1902. Notes on Cambrian faunas. Transactions of the Royal Society of Canada, 8 (4), 93-112.

Montero, A. 1995. La colección de invertebrados fósiles del Museo Nacional de Ciencias Naturales. Su trayectoria histórica y museológica. Tesis Doctoral, Universidad Complutense de Madrid. 807 pp. [Inédita.]

Montero, A. and Diéguez, C. 1991a. Rehousing of paleontological collections in the Museo Nacional de Ciencias Naturales, Madrid, Spain. Collection Forum, 7 (1), 10-12.

Montero, A. y Diéguez, C. 1991b. Tipología, problemática y usos de la documentación asociada a colecciones paleontológicas. Boletín de la Asociación Nacional de Archiveros, Bibliotecarios, Museólogos y Documentalistas, 41 (2), 153-162.

Montero, A. y Diéguez, C. 1996. Fauna de Burgess Shale (Cámbrico Medio) en el Museo Nacional de Ciencias Naturales-C.S.I.C., Madrid, España. In: Comunicaciones de las XII Jornadas de Paleontología: Badajoz, 30 de octubre-2 de noviembre de 1996. (Eds. T. Palacios y R. Gozalo). Universidad de Extremadura, Servicio de Publicaciones, Cáceres, 83-84.

Walcott, C. D. 1912. Cambrian geology and paleontology, II. No. 6.-Middle Cambrian Branchiopoda, Malacostraca, Trilobita and Merostomata. Smithsonian Miscellaneous Collections, 57 (6), 145-228.

Walcott, C. D. 1916. Cambrian geology and paleontology, III. No. 5.-Cambrian Trilobites. Smithsonian Miscellaneous Collections, 64 (5), 301-456.

Walcott, C. D. 1919. Cambrian geology and paleontology, IV. No. 5.-Middle Cambrian Algae. Smithsonian Miscellaneous Collections, 67 (5), 217-260.

Walcott, C. D. 1920. Cambrian geology and paleontology, IV. No. 6.-Middle Cambrian Spongiae. Smithsonian Miscellaneous Collections, 67 (6), 261-364.

Walcott, C. D. 1924. Cambrian geology and paleontology, IV. No. 10.-Cambrian and Ozarkian Brachiopoda. 
Smithsonian Miscellaneous Collections, 67 (10), 477. 554.

Westergård, A. H. 1936. Paradoxides oelandicus beds of Öland, with an account of diamond boring through the Cambrian of Mossberga. Sveriges Geologiska Undersökning, Series C, 394 (Årsbok 30, n 1), 1-66.
Whittington, H. B. 1985. The Burgess Shale. Yale University Press, New Haven, London. 151 pp.

Yochelson, E. L. 1996. Discovery, collection, and description of the Middle Cambrian Burgess Shale biota by Charles Doolittle Walcott. Proceedings of the American Philosophical Society, 140 (4), 469-545. 